\title{
Isolated fallopian tube torsion in an adolescent: a diagnostic challenge
}

\section{Leonor Bivar*, Ana Castro, Neusa Teixeira}

Department of Gynaecology and Obstetrics, Hospital de Braga, Braga, Portugal

Received: 26 October 2020

Accepted: 04 December 2020

\section{*Correspondence:}

Dr. Leonor Bivar,

E-mail: leonorbivar@gmail.com

Copyright: (c) the author(s), publisher and licensee Medip Academy. This is an open-access article distributed under the terms of the Creative Commons Attribution Non-Commercial License, which permits unrestricted non-commercial use, distribution, and reproduction in any medium, provided the original work is properly cited.

\begin{abstract}
Torsion of the fallopian tube without ovarian involvement is an extremely rare event and often difficult to diagnose, but noteworthy, cause of lower abdominal pain. The authors describe a case related to a 14- year-old adolescent with acute lower abdominal pain and vomiting presented to the gynaecological emergency department. Blood tests exhibited modest biochemical parameters of inflammation and a transvaginal ultrasound showed a left adnexal tubular cystic mass with normal ovarian appearance. A diagnostic laparoscopy revealed an isolated fallopian tube torsion. The tube was able to be untwisted with restoration of pelvic anatomy and the follow-up was uneventful. The authors describe a challenging diagnosis in an adolescent in which delayed intervention could compromise her reproductive future. Tubal torsion should however be kept in mind in the differential diagnosis of lower abdominal pain in women of all ages.
\end{abstract}

Keywords: Isolated tubal torsion, Gynaecology surgery, Pediatric

\section{INTRODUCTION}

Adnexa torsion is an infrequent cause of pain in the lower abdomen. However, the event is a common gynaecologic surgical emergency, with a prevalence of $2.7 \% .^{1}$ Fallopian tube torsion, while far less common than adnexal torsion, may present in a remarkably similar fashion or with fewer findings. As the clinical findings are nonspecific and unimpressive with no reliable ultrasound signs, this diagnosis can be difficult to suspect. Recognition is therefore often delayed causing irreversible ischaemic damage, and ultimately peritonitis, specially concerning among young females that have not conclude their family project.

We report a rare case of isolated fallopian tube torsion in a young female patient, pretending to highlight the need for high suspicion index, early diagnosis and prompt clinical decision and intervention.

\section{CASE REPORT}

A 14-year-old female, with irrelevant medical or family history, presented to the Gynaecological Emergency Department with persistent sudden onset lower abdominal pain located in the left iliac fossa, with 10 hours of evolution, and vomiting. There were no associated fever and urinary or bowel symptoms. The patient never had abdominal surgery and denied being sexually active, referring regular menses, despite unable to recall the date of her last menstrual period. She was poorly cooperative due to pain complains.

On admission, the patient was hemodynamically stable, with blood pressure of 112/64 $\mathrm{mmHg}$ and pulse rate of 75 ppm, and without fever. On physical examination, the abdomen was soft, and palpation was painful, with slight guarding sign on the left iliac fossa. There were no palpable masses. On gynaecological examination, the hymen appeared to be intact and complacent despite not allowing bimanual examination; however there seems to be no relevant change on vaginal discharge. Transvaginal 
ultrasound, performed after verbal consent of both patient and father, reveal a well-defined tubular cystic mass in left adnexal region with $63.5 \times 21.8 \mathrm{~mm}$ (Figure 1) and a small amount of fluid on pouch Douglas; ipsilateral ovary and contralateral adnexa shows no abnormalities. The color doppler was inconclusive. Blood biochemistry reported mild leucocytosis, white blood cells (WBC) $15.3 \times 103 / \mu 1$ (normal 4.0-13.5×103/ $\mu 1$ ), neutrophilia $9.6 \%$ and a marginally elevated C-reactive protein (CRP) (12.0 $\mathrm{mg} / \mathrm{L}$, normal $<5.0 \mathrm{mg} / \mathrm{l})$. The sediment urinary test was normal, and the pregnancy test was negative.

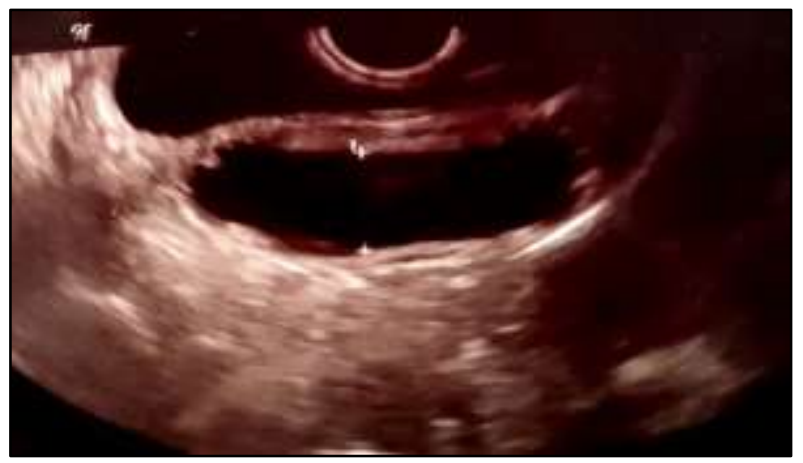

Figure 1: Tubular cystic mass in left adnexal region with $63,5 \times 21,8 \mathrm{~mm}$.

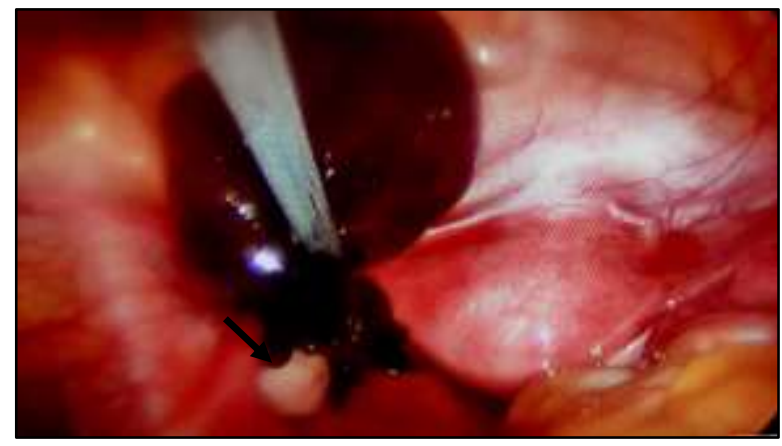

Figure 2: Oedematous left tube with a bluish-black appearance and a normal ipsilateral ovary (arrow).

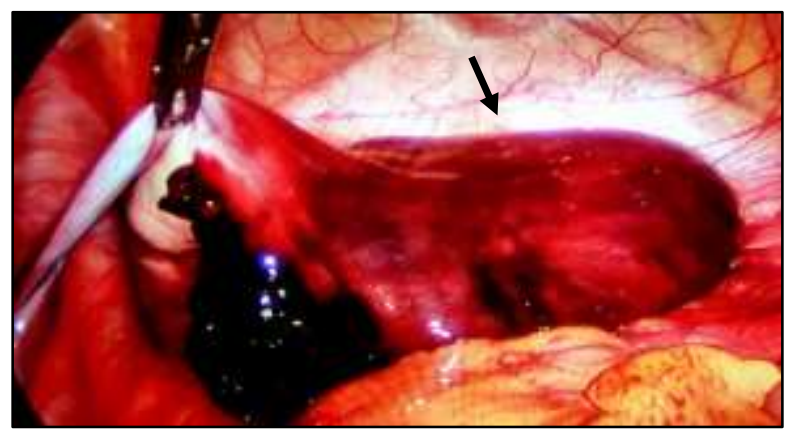

Figure 3: Tubal appearance suggesting revascularization after detorsion (arrow).

This case required a challenging process of diagnostic and therapeutic decision-making. Since the patient was in reproductive age, and because left laterality makes it less probable to be an acute appendicitis, initial concern was to exclude an ectopic pregnancy. The presence of her father, that could preclude the patient from revealing sexual activity, and the fact of not being able to recall the date of her last menstrual period contributed to our apprehension. Considering the left tubular cystic lesion and blood tests, hydrosalpix or inflammatory pelvic disease may be suspected. However, in a patient without sexual activity upper gynaecological tract infection is very unlikely. Or could it be an adnexal torsion? Corroborating this hypothesis, the insidious presentation with pain refractory to analgesia associated with a left adnexal mass; however, the ipsilateral ovary was normal on ultrasound. Therefore, the hypothesis of isolated fallopian tube torsion was raised as an exclusion diagnosis.

A diagnostic laparoscopy was performed and revealed a large oedematous left tube with a bluish-black appearance twisted at the cornual end (Figure 2). There was no ipsilateral ovarian involvement. The uterus, right adnexa and the rest of the peritoneal cavity were investigated but no abnormalities were detected. The tube was able to be untwisted laparoscopically with gradual return of blood flow (Figure 3). No necrosis, gelatinous tissue or bleeding were noted, and so salpingectomy was not performed, allowing conservative treatment.

The patient made an uneventful recovery with home discharge by postoperative day two and was referred to a pediatric appointment for follow-up.

\section{DISCUSSION}

In 1980, Bland-Sutton was the first one reporting isolated fallopian tube torsion. ${ }^{2}$ It is a rare gynaecological cause of acute lower abdominal pain, affecting one in 1.500000 women. ${ }^{3}$ The majority of adnexal torsion cases occur in women of reproductive age, because almost all the risk factors are rare before menarche or during menopause. ${ }^{3}$ However, it has been reported in females of all ages, including pediatric patients and even in pregnancy. ${ }^{4}$ Etiologic factors have been described and classified as either internal or external. Internal factors include congenital anomalies, acquired pathology and abnormal peristalsis. The latter category includes conditions affecting the neighbouring organs, venous congestion and medication. ${ }^{5}$

Isolated fallopian tube torsion can present without any obvious pathognomonic symptoms and clinical or radiological signs. The most common symptom is lower abdominal pain, most frequently of sudden onset and accompanied by nausea, vomiting or urinary urgency; fever may be a marker of adnexal necrosis, particularly in the setting of leukocytosis. ${ }^{4}$

A presumptive diagnosis of torsion can be made with a fair degree of confidence in the presence of acute pelvic pain and an adnexal mass, after exclusion of major 
surgical emergencies. The imaging characteristics on ultrasound may include a variably thickened tubular structure in a folded configuration or a cystic adnexal mass. There may be debris seen within the fallopian tube, compatible with hematosalpinx. ${ }^{6}$ It has been suggested that color Doppler interrogation of the tube may demonstrate high impedence arterial flow in the wall of the mass with reversed diastolic flow or no demonstrable vascular flow. ${ }^{6}$ However, the persistence of arterial flow does not rule out adnexal torsion. ${ }^{7}$ This may be due to the dual blood supply to the adnexa, to the intermittent or partial torsion, or to the venous occlusion occurring before arterial obstruction. ${ }^{7}$ Utility of color Doppler sonography may be in determining the preoperative viability of the ovary or fallopian tube.

The mainstay of treatment of adnexal torsion is prompt operative evaluation to preserve ovarian function and prevent other adverse effects (hemorrhage, peritonitis, adhesion formation). Historically, salpingooophorectomy had been the standard treatment of a torsed ovary because it was believed that conserving the ovary would leave behind necrotic tissue and restoring normal anatomy via detorsion could dislodge a clot in the ovarian vein and result in an embolus. ${ }^{8}$ However, it appears that detorsion is associated with continued ovarian function in many women and the key factor is to perform it as quickly as possible. ${ }^{9}$ The standard approach to determining tissue viability is gross visual inspection. Rarely, ovarian or tubal necrosis is present at time of surgery. The appearance on gross inspection of a necrotic ovary or tube includes a loss of normal anatomic structure and a gelatinous or poorly defined structure. The ability to retain viability despite prolonged ischaemia, as revealed by the good outcome in apparently severely injured ovaries reported in literature, indicates that complete arterial obstruction does not usually occur, and some blood supply can still be obtained from either the ovarian or uterine arteries. ${ }^{9}$

\section{CONCLUSION}

In conclusion, since there are no clinical pathognomonic features, there is often a delay in diagnosis of isolated fallopian tube torsion, with the ultimate diagnosis often occurring in the operating room. Imaging can sometimes be helpful to propose this difficult diagnosis and should, at least, be considered as a possibility with a suggestive clinical history in conjunction with a normal ipsilateral ovary with persistence of arterial flow. Prompt intervention may allow for laparoscopic restoration of pelvic anatomy, rather than salpingectomy, which can help preserve fertility in a young patient.

Funding: No funding sources

Conflict of interest: None declared

Ethical approval: Not required

\section{REFERENCES}

1. Sanfilippo JS, Rock JA. Surgery for benign disease of the ovary. In: TeLinde's Operative Gynecology, 11 th ed., Jones HW, Rock JA (Eds), Wolters Kluwer. 2015.

2. Bland-Sutton J. Salpingitis and some of its effects. Lancet 2. 1890;1146-8.

3. Comerci G, Colombo FM, Stefanetti M, Grazia G. Isolated fallopian tube torsion: a rare but important event for women of reproductive age. Fertil Steril. 2008;90(4):1198.e23-5.

4. Tsafrir Z, Hasson J, Levin I, Soloman E, Lessing JB et al. Adnexal torsion: cystectomy and ovarian fixation are equally important in preventing recurrence. Eur J Obstet Gynecol Reprod Biol. 2012;162:203-5.

5. Ziogas AC, Thanasas IK, Oikonomou IT, Tsiamanta C. Torsion of the left fallopian tube without ovarian involvement in a 47-year-old woman: A case report. Case Reports in Women's Health 26. 2020; e00179.

6. Richard HM, Parsons RB, Broadman KF, Shapiro RS, Yeh HC. Torsion of the fallopian tube: progression of sonographic features. $\mathrm{J}$ Clin Ultrasound. 1998;26:374-6.

7. Ssi-Yan-Kai G, Rivain AL, Trichot C, Morcelet MC, Prevot S, Deffieux $X$ et al. What every radiologist should know about adnexal torsion. Emerg Radiol. 2018;25(1):51-9.

8. Jones HW, Jones GS. Novak Textbook of Gynecology, 10th ed, Wolters Kluwer Lippincott Williams \& Wilkins, Baltimore. 1981;471.

9. Oelsner G, Cohen SB, Soriano D, Admon D, Masiach S, Carp H. Minimal surgery for the twisted ischaemic adnexa can preserve ovarian function. Hum Reprod. 2003;18:2599.

Cite this article as: Bivar L, Castro A, Teixeira N. Isolated fallopian tube torsion in an adolescent: a diagnostic challenge. Int J Reprod Contracept Obstet Gynecol 2021;10:340-2. 